\title{
UN CAS hUMAIN DE FASciolase hÉpatique
}

\section{Par FERNANDO DA FONSECA et J. FRAGA DE AZEVEDO}

Selon Brumpt, jusqu'en 1931, la littérature médicale signalait seulement 108 cas humains de fasciolase. Le même auteur en 1936 (Précis de Parasitologie, 5 $5^{\circ}$ édition, 1936) s'occupe de travaux récents, montrant la fréquence relative de cette infection au Venezuela, en France, en Algérie, en Italie, en Arménie, au Turkestan, en Egypte, au Chili et en Argentine. Belding (Textbook of Clinical Parasitology, 1942) rapporte qu'au moins 130 cas ont été enregistrés dans la littérature médicale. Kouri (Clinical Laboratory Methods and Diagnosis : Gradwohl, London 1943) considère l'infection par Fasciola hepatica comme un problème de la plus grande importance à Cuba, où les 2 premiers cas autochtones ont été décrits par cet auteur et Arenas en 1931, et où, en avril 1942, le nombre de cas était déjà supérieur à 40 .

Mackie, Hunter et Worth (A Manual of Tropical Medicine prepared under the auspices of the Division of Medical Sciences of the National Research Council, Philadelphia and London, 1945) ont présenté en 1945 le tableau suivant sur la distribution de la fasciolase humaine :

\begin{tabular}{|c|c|c|}
\hline $\begin{array}{l}\text { AFrIQUE : } \\
\text { Algérie. } \\
\text { Soudan Français. } \\
\text { AsıE : } \\
\text { Chine. } \\
\text { Turkestan. } \\
\text { AsIE MINEurE : } \\
\text { Syrie. } \\
\text { Turquie. } \\
\text { AustraliE : } \\
\text { Queensland. }\end{array}$ & $\begin{array}{l}\text { EuRoPE : } \\
\text { Corse. } \\
\text { France. } \\
\text { Italie. } \\
\text { Hongrie. } \\
\text { Roumanie. } \\
\text { Salonique. } \\
\text { Ecosse. } \\
\text { U.R.S.S. }\end{array}$ & $\begin{array}{l}\text { AmÉRIQUe : } \\
\text { Argentine. } \\
\text { Chili. } \\
\text { Porto-Rico. } \\
\text { Cuba. } \\
\text { Vénézuéla. }\end{array}$ \\
\hline
\end{tabular}

AnN. de Parasitologie, T. XXIII, Nos $1-2$. -1948 , p. 18-22. 
Au Portugal continental et dans les iles adjacentes, nous ne connaissons pas de publications sur la fasciolase humaine. Notre malade, naturel du Funchal (île de Madère), nous a fait le récit suivant :

Observation. - 3-XI-1947. - A. V. F., 31 ans, marié, employé de commerce, habitant à Funchal, oủ il a toujours vécu. Il vient pour la première fois sur le continent.

Dans le passé il signale une fièvre typhoïde, une blennorragie et des chancres mous.

Il y a environ un an qu'il est sujet à des perturbations intestinales vagues, avec deux selles quotidiennes, une le matin et l'autre à une heure variable, quelquefois après le repas de midi. Les selles sont pâteuses et parfois liquides, sans mucosités, ni sang, ni lientérie.

Il se plaint de douleurs dans le dos, d'abattement, de nervosité, de franspirations nocturnes, et signale que parfois il dort mal. De temps en temps, il se sent l'estomac lourd, même après des repas légers. Habituellement il ne suit pas de régime et mange fréquemment du cresson et de la laitue crus. Il se sent plus mal quand il mange des fruits crus.

L'observation révèle un individu de type asthénique, un peu amaigri, le ventre ballonné. La langue est humide et légèrement pâteuse. Le ventre est douloureux au toucher, surtout le trajet du côlon. Le rebord du foie est en situation normale, un peu dur et douloureux. Les examens radiologiques faits à l'estomac et aux intestins ont révélé que la rate est augmentée de volume.

Ailleurs l'observation est négative.

12-XI-1947. - Depuis hier soir le malade présente aux mains, au cou et au ventre une éruption urticariforme accompagnée de prurit.

Examens de Laboratoire

Sang

\begin{tabular}{|c|c|c|c|}
\hline RXAMEN CYTOLOGIQUE & $11-X I$ & $17-\mathrm{XI}$ & $26-X I$ \\
\hline Globules blanes par $\mathrm{mm}^{3}{ }^{3} \ldots$ & 8.100 & & 8.100 \\
\hline Granulocytes neutrophiles... & 36 & 385 & 25,5 \\
\hline - éosinophiles... & 40,5 & 34,5 & 49,5 \\
\hline basophiles..... & 1,0 & 1,0 & 0,0 \\
\hline Lymphocytes ............... & 15,5 & 18,5 & 19,5 \\
\hline Monocytes ................ & 7,0 & $\begin{array}{r}0,0 \\
7.5\end{array}$ & 5,5 \\
\hline Globules rouges par $\mathrm{mm} .^{3} \ldots \ldots$ & 4.400 .000 & & $\begin{array}{l}4.170 .000 \\
870.100\end{array}$ \\
\hline Hémoglobine (Sahli) . ......... & 86 p. 100 & & (1. 104 \\
\hline Valeur globulaire ............. & 0,97 & & \\
\hline
\end{tabular}


On n'a pas noté d'altérations appréciables dans la morphologie des globules rouges. Le pourcentage de réticulocytes détermuní le 26 novembre a été de $2,2 \%$.

Réactions de Wassermann et de Kahn: négatives.

Réaction de Hanger (céphaline-cholestérol): après 24 heures : négative ; après 48 heures : très faiblement positive (+ -$)$.

Réaction de Takata-Ara: négative.

Selles: on a fait des examens microscopiques des selles les 19, $20,22,24$ et 26 novembre. Les selles des 19 et 20 ont été recueillies au laboratoire et examinées immédiatement après leur émission dans l'étude de Foot à $37^{\circ}$. Leur examen a révélé des formes végétatives d'Entamœba dysenteriæ. Dans tous les échantillons de selles examinés, se trouvent des œufs de Fasciola hepatica, Ascaris lumbricoides et Trichocephalus dispar.

Dans les selles recueillies le 25 novembre, on a procédé à la recherche des helminthes, après un sondage duodénal, mais le résultat a été négatif.

Bile : on a recueilli la bile à deux reprises (22 et 26 novembre), en procédant à un sondage duodénal avec sulfate de magnésium. On a toujours obtenu les trois types de bile A, B et C. L'examen du sédiment de centrifugation des trois échantillons a révélé des œufs de Fasciola hepatica, seulement dans la bile C recueillie le 25.

Examen radiologique de l'estomac, des intestins et de la rate: il montre une accélération de la circulation intestinale et une légère splénomégalie.

Diagnostic : l'infestation humaine par Fasciola hepatica se déroule le plus souvent avec une grande pauvreté de symptômes et ceux-ci orientent davantage le praticien vers la maladie gastro-intestinale. Le tableau habituel est celui des perturbations dyspeptiques gastriquies : douleurs sur tout le trajet du côlon et crises alternées de constipation et de diarrhée. La symptomatologie hépato-biliaire, moins fréquente, va de la simple douleur sourde dans la région hépatique, avec irradiation dans le dos, jusqu'à la colique hépatique répétée avec ou sans ictère et parfois accompagnée de fièvre. Le déroulement, souvent chronique, amène chez certains malades des troubles mentaux. Quelques cas de Kouri, avant la découverte de leur nature parasitaire, ont été diagnostiqués : cholécystite, colique hépatique, ictère par obstruction, colique appendiculaire, ulcère duodénal, fièvre bilio-septique, hépatite amibienne aiguë et malaria. ria.

Dans notre cas, l'éosinophilie a été le fil conducteur, car, étant 
donné l'absence de symptômes qui aient pu permettre de penser aux maladies qui donnent le plus fréquemment un pourcentage si élevé d'éosinophiles, un examen des selles s'imposait, en vue d'éclaircir le problème. La crise d'urticaire observée chez notre malade est citée dans la fasciolase par plusieurs auteurs.

Le fait que nous avons trouvé des œufs caractéristiques dans plusieurs examens de selles, faits ì quelques jours d'intervalle, ainsi que dans la bile $\mathrm{C}$, recueillie par sondage duodénal, rend indubitable le diagnostic de l'infestation du foie par Fasciola hepacita. En vérité, on doit tenir compte que l'apparition d'œufs de cet helminthe dans les selles peut-être en relation avec l'ingestion de foie de mouton ou d'un autre animal (bœuf, porc, cheval, etc...) infecté par ce parasite. Les œufs trouvés correspondront, cependant, à un véritable parasitisme si, plusieurs jours avant l'examen des selles, le malade n'a pas consommé de foie d'un des animaux cités, et si la recherche est positive dans la bile et les échantillons de selles recueillis en des jours divers, comme il advint en notre cas. Nous devons rapporter aussi que les œufs trouvés étaient d'aspect typique : jaunâtres, ovoïdes, operculés, à coque mince, non embryonnés et ayant, par la mesure de 5 œufs, en moyenne $132 \mu \times$ $77 \mu(128$ à $135 \mu \times 67$ à $81 \mu)$.

La seule présence, dans les selles du malade, de formes végétatives d'Entamioba dysenteriæ et d'œufs d'Ascaris lumbricoides et de Trichocephalus dispar, conduit évidemment au diagnostic d'infestation concomitante par ces parasites.

Mode d'infection : considérant que l'homme peut s'infecter avec la Fasciola hepatica seulement par l'ingestion de métacercaires qui, comme on le sait, peuvent se trouver soit adhérentes aux plantes aquatiques, soit libres dans l'eau même, et considérant que notre malade mangeait fréquemment du cresson, il est à présumer que cette plante aquatique a été le véhicule de la forme larvaire infectante. Etant donné que selon ses informations, cette plante aquatique est consommée fréquemment dans l'île de Madère, par sa population, il est à présumer que d'autres cas humains de Fasciola hepatica y surviennent. Ce soupçon justifie entièrement qu'une étude minutieuse de la question y soit faite, ce que nous recommandons à la Société portugaise de Biologie et aux médecins de Funchal.

Pour compléter l'étude de notre cas, nous tentons maintenant de provoquer l'évolution du parasite à partir des œufs éliminés avec 
les selles. $\mathrm{Si}$, à partir de ces œufs, nous réussissons à établir complètement le cycle de Fasciola hepatica, nous pourrons conclure que l'organisme humain n'a été en rien défavorable à sa vitalité, qu'il est par conséquent favorable à son évolution, ce qui revient à dire que l'homme, atteint par les métacercaires, a toutes chances de contracter l'infection par le parasite. Nous rendrons compte ultérieurement du résultat de nos investigations.

Traitement: parmi les divers médicaments conseillés pour la fasciolase, nous avons choisi le chlorhydrate d'émétine, mettant ainsi à profit également son action amibicide spécifique. Nous attendons le résultat de cette thérapeutique.

Institut de médecine tropicale de Lisbonne. 